Original Research

\title{
Bone Mineral Density in Male Hospital Physicians over the Age of 65 Years
}

Noa Sylvetsky ${ }^{1}$, Chen Futeran Shahar ${ }^{2}$, Meir Frankel $^{1,}{ }^{*}$, Gabriel Munter ${ }^{1}$

1. Department of Internal Medicine and Endocrine Unit, Shaare Zedek Medical Center, Jerusalem, Israel, affiliated with the Faculty of Medicine, Hebrew University, Jerusalem, Israel; E-Mails: noas@szmc.org.il; meirf@szmc.org.il; gmunter@szmc.org.il

2. Department of Obstetrics and Gynecology, Shaare Zedek Medical Center, Jerusalem, Israel, affiliated with the Faculty of Medicine, Hebrew University, Jerusalem, Israel; E-Mail: chen.futeran@gmail.com

* Correspondence: Meir Frankel; E-Mail: $\underline{\text { meirf@szmc.org.il }}$

Academic Editor: Ray Marks

Special Issue: Osteoporosis in the Elderly

OBM Geriatrics

2020, volume 4, issue 3

doi:10.21926/obm.geriatr.2003126
Received: April 01, 2020

Accepted: June 22, 2020

Published: July 02, 2020

\begin{abstract}
Hospitalists are at risk for vitamin D deficiency during their active years due to indoor working conditions and insufficient sunlight exposure. The impairment of bone mineral density (BMD) in this group has not been studied. A total of 50 male hospitalists aged $\geq 65$ years were enrolled. Their BMD was measured at the femoral neck (FN), lumbar spine (LS), and distal radius (DR), and their medical history and risk factors were assessed through a detailed questionnaire. The FRAX (Fracture risk assessment tool) score was calculated for each participant. The mean age was $71 \pm 5.3$ years. They worked as hospital physicians for a mean duration of $38.8 \pm 6.9$ years. According to the BMD measurement, 15 (30\%) had osteoporosis, and 29 (58\%) had osteopenia. We also analyzed bone density excluding DR, since the clinical significance of low bone density of DR alone is debatable. In this analysis, 7 doctors (14\%) had osteoporosis, and 33 (66\%) had osteopenia. According to AACE/ACE 2016 guidelines, $48 \%$ of the participants would require specific treatment for fracture prevention.
\end{abstract}

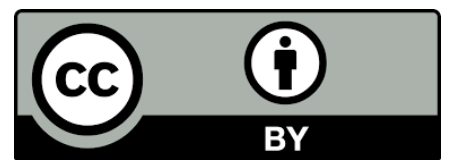

(c) 2020 by the author. This is an open access article distributed under the conditions of the Creative Commons by Attribution License, which permits unrestricted use, distribution, and reproduction in any medium or format, provided the original work is correctly cited. 
However, using only FN and LS BMD, 40\% would require treatment. Hospitalists were found to have a high prevalence of osteoporosis and osteopenia, and $40 \%$ required specific treatment according to international guidelines.

\section{Keywords}

Osteoporosis; osteopenia; bone mineral density; male physician; hospitalist

\section{Introduction}

Severe vitamin D deficiency in childhood is known to cause rickets; however, this is rare in the developed world, in contrast to mild to moderate vitamin $D$ deficiency that frequently occurs across all age groups [1]. Vitamin D deficiency contributes to the development of osteoporosis and osteomalacia and is associated with an increased risk of bone fractures in the elderly, bonerelated pain, diabetes mellitus [2], cardiovascular disease [3], and an increase in cancer mortality [4], whereas vitamin D supplementation is associated with decreased total mortality rate [5].

Solar ultraviolet-B irradiation is the primary source of vitamin D for most people, while dietary sources are limited. Therefore, sunlight exposure is an important factor in serum 25(OH)D levels. Exposure varies seasonally, and certain populations are not exposed to sunlight due to limited mobility or traditional dress. In recent years, many large observational studies have found evidence of vitamin D deficiency, even in young and healthy subjects. Vitamin D inadequacy was reported in approximately $36 \%$ of otherwise healthy adults, in up to $57 \%$ of general medicine inpatients in the United States and even higher in Europe [1]. Even in sunny countries such as Israel, hypovitaminosis D is prevalent. In a study published in 2009, four consecutive measurements of vitamin D, were taken in outdoor and indoor Israeli workers, once per season. Among this working population, optimal vitamin $D$ status ( $\geq 75 \mathrm{nmol} / \mathrm{L})$ was achieved only in summer by males working either outdoors or indoors [6].

Hospitalists are at-risk for vitamin D deficiency due to long hours of indoor work with little sun exposure $[7,8]$.

In a previous study performed in our institution [9], vitamin D levels of 51 young hospitalists were compared to those of young community-based physicians, at the end of winter, and the mean serum level of $25(\mathrm{OH}) \mathrm{D}$ among the hospital physicians was found to be significantly lower than that of community-based physicians.

In a recent systematic review on vitamin D levels in different occupations [10], the overall mean serum 25(OH)D levels of all healthcare workers were $61.6 \pm 11.0 \mathrm{nmol} / \mathrm{L}$ (data from 19,083 study subjects from 35 studies). Among healthcare workers, medical residents and healthcare students had the lowest levels of circulating vitamin $D(44.0 \pm 8.3 \mathrm{nmol} / \mathrm{L}$ and $45.2 \pm 5.5 \mathrm{nmol} / \mathrm{L}$, respectively). Up to $95 \%$ of healthcare workers had vitamin D insufficiency.

In our study, we examined hospital physicians and pensioners, a group at risk for low vitamin D levels in their younger years, to explore the possible effects of this deficiency on bone density after several years. Osteoporosis is more common in women than in men and has therefore been studied more extensively in women. However, osteoporosis in men is an important public health problem with associated morbidity and mortality at a significant level [11]. 


\section{Materials and Methods}

The study was conducted in the Shaare Zedek Medical Center (SZMC), a 1000-bed teaching hospital in Jerusalem. The study group consisted of male hospital physicians aged 65 and older, who were invited to participate through email and telephone.

We excluded doctors who did not consistently work in a hospital during their careers, were currently receiving chronic steroid treatment, had malabsorption, chronic kidney disease with GFR<60, osteoarthritis, a current cancer diagnosis that involved endocrinological abnormalities or treatment that could affect bone density (including androgen-deprivation therapy), and doctors with known osteoporosis [12].

Osteopenia and osteoporosis were defined according to the World Health Organization (WHO) criteria [13].

\subsection{Protocol}

Each participant filled in a questionnaire detailing age, the field of medical specialty, number of years having worked as a hospital doctor, and status as a Holocaust survivor, as well as his medical history regarding the exclusion criteria and FRAX ${ }^{\circledast}$ (Fracture risk assessment tool) variables.

For Bone Mineral Density (BMD) measurement, all participants were tested using the dualenergy x-ray absorptiometry (DXA) device-a Hologic QDR Series X-Ray Bone Densitometer. The $B M D$ was measured at the femoral neck (FN), lumbar spine (LS), and distal radius (DR). All subjects were tested by the same experienced technician, and the lumbar vertebrae were marked manually.

FRAX ${ }^{\circledast}$ score was calculated for each participant using FRAX ${ }^{\circledast}$ ISRAEL data at the Sheffield University website [14]. It is a fracture risk assessment tool that has been developed by Sheffield University and estimates the 10-year probability of hip fracture and major osteoporotic fracture, in order to help health care professionals identify patients who need pharmacologic treatment. It is based on data collected from large observational studies and global population. The FRAX ${ }^{\otimes}$ score calculation includes age, gender, weight, height, clinical risk factors (previous fracture, parent hip fracture, current smoking, glucocorticoid use, rheumatoid arthritis, secondary osteoporosis, and alcohol use), and femoral neck BMD $\left(\mathrm{g} / \mathrm{cm}^{2}\right)$. The $F R A X^{\circledast}$ score prediction accuracy has been validated in many independent studies [15].

The institutional review board of Shaare Zedek Medical Center provided approval for this study. Each participant signed an informed consent form.

\subsection{Statistical Methods}

Demographic and clinical characteristics were analyzed descriptively as numbers and percentages or means and standard deviations, as appropriate.

To test the association between two categorical variables, the Chi-square test was applied. Pearson's correlation coefficient was calculated to assess the correlation between two quantitative variables. A P-value of $5 \%$ or less was considered statistically significant.

The SPSS 21.0 statistical software package (SPSS Inc., Chicago, IL, U.S.A.) was used to perform the statistical analysis. 


\section{Results}

Out of the 110 physicians invited to participate in the study, 8 (7\%) were excluded according to the exclusion criteria, and $52(46 \%)$ declined to participate. A total of 50 physicians were included in the final statistical analysis. Their characteristics are shown in Table 1 . The mean age was $71 \pm 5.3$ years, and they worked as hospital physicians for $38.8 \pm 6.9$ years. There were $15(30 \%)$ who had osteoporosis at one of the sites measured, 29 (58\%) had osteopenia, and $6(12 \%)$ had normal bone density (Figure 1). We also analyzed bone density excluding DR, since the clinical significance of low bone density of DR alone is debatable (Figure 1). In this analysis, 7 doctors (14\%) had osteoporosis, 33 (66\%) had osteopenia, and 10 (20\%) had normal bone density.

Table 1 Participants' characteristics $(\mathrm{N}=50)$.

\begin{tabular}{|l|c|}
\hline Age (years, mean \pm SD) & $71 \pm 5.3$ \\
\hline Years as a hospital physician (years, mean \pm SD) & $38.8 \pm 6.9$ \\
\hline BMI (mean) & 26.3 \\
\hline Medical History & $1(2 \%)$ \\
\hline Renal Failure & $3(6 \%)$ \\
\hline Cancer (past) & $2(4 \%)$ \\
\hline Smoker & $5(10 \%)$ \\
\hline Holocaust survival & $1(2 \%)$ \\
\hline $\begin{array}{l}\text { Steroid treatment } \\
\text { (present or past) }\end{array}$ & $2(4 \%)$ \\
\hline Hyperthyroidism & $2(4 \%)$ \\
\hline S/P Gastrectomy & \\
\hline
\end{tabular}

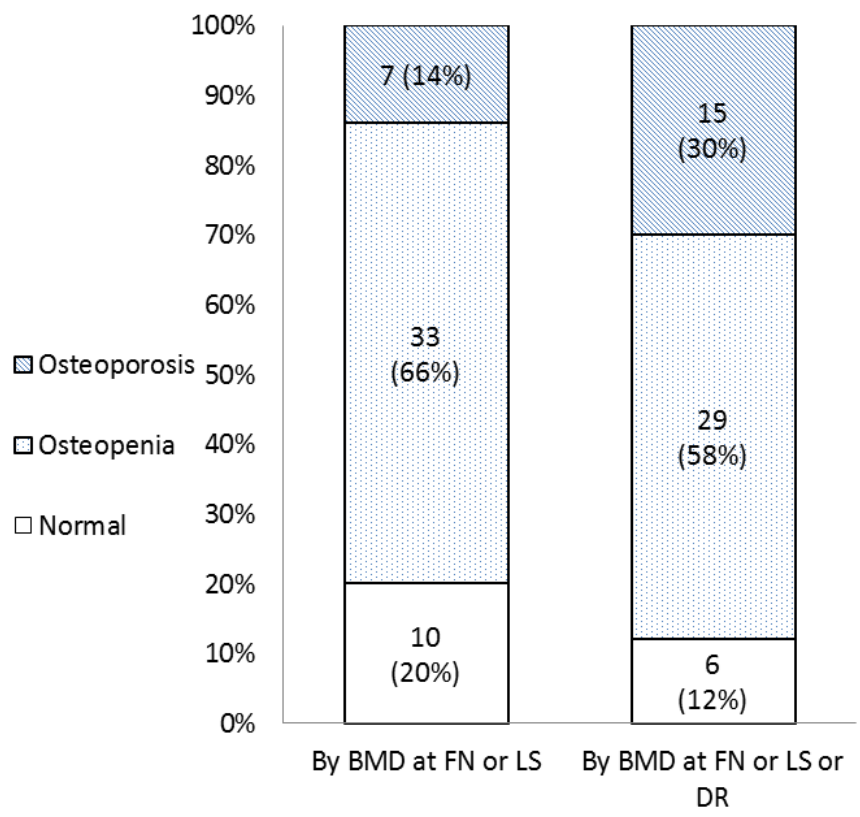

Figure 1 Diagnosis by BMD. BMD: Bone Mineral Density; FN: Femoral Neck; LS: Lumbar Spine; DR: Distal Radius. 
The FRAX ${ }^{\oplus}$ score was calculated for each participant, and we used AACE/ACE 2016 guidelines to define the participants who required specific therapy for fracture prevention, i.e., a participant who had osteoporosis at one of the sites or osteopenia with a high FRAX ${ }^{\circledR}$ score (Figure 2). This analysis showed that $48 \%$ of the participants would require specific pharmacologic treatment for fracture prevention. However, $40 \%$ would require treatment, using only FN and LS BMD.

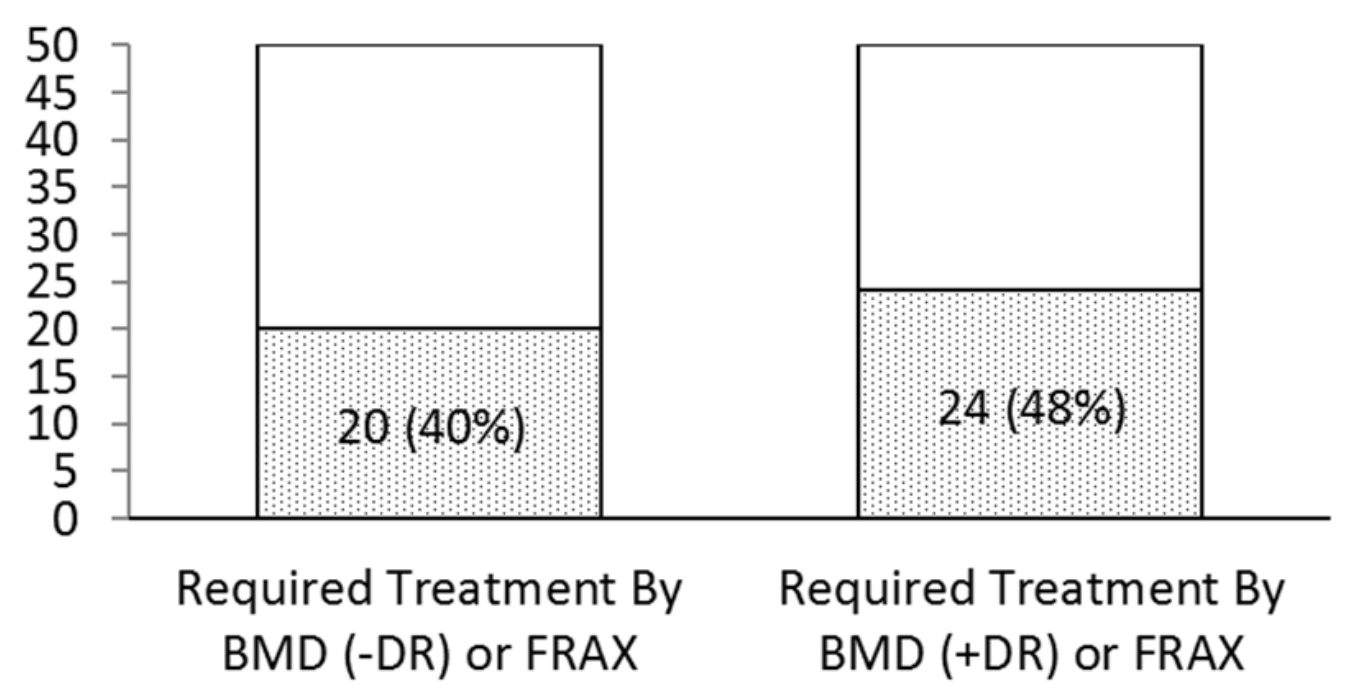

Figure 2 Required metabolic treatment by BMD result and/or FRAX score. BMD (-DR): Bone Mineral Density at Femoral Neck or Lumbar Spine; BMD (+DR): Bone Mineral Density at Femoral Neck or Lumbar Spine or Distal Radius.

\section{Discussion}

The prevalence of osteoporosis or osteopenia in hospital physicians over the age of 65 , was found to be $30 \%$ and $58 \%$, respectively. It might be explained, partly by vitamin D deficiency, which was observed in a similar population in a previous study. Another factor that can influence BMD in this population is the low level of physical activity due to heavy workload.

The use of BMD as a screening test for men is debatable. Certain expert groups recommend screening for men aged 70 years and older [16, 17], or 65 and older [18], while others do not recommend screening $[19,20]$. Although about $30 \%$ of the hip [21] and $33 \%$ of vertebral fractures occur in males [22], most men do not undergo BMD testing, and considerably less epidemiological data are available for men than women.

There is no data on the presence of osteopenia and osteoporosis in the Israeli male population to directly compare the study results. The data on the prevalence of osteopenia or osteoporosis in men worldwide are limited. Most of the data are derived from the National Health and Nutritional Examination Survey (NHANES), which is published periodically and from the MrOS study. The NHANES survey in the US in 2005-2010 found osteoporosis in 5\% of men at the ages 70-79 and osteopenia in $41.8 \%$, using FN and LS BMD [23]. In our study, the prevalence of osteoporosis was $5 \%$ and of osteopenia $85 \%$ in the age group of 70-79 years (using FN and LS BMD). The prevalence of osteopenia in our study was higher than the earlier reported prevalence, while the mean age in our study was 71 and only 5 doctors were 80 years and older. The MrOS study [24] found osteoporosis in $3.3 \%$ of men older than 65 years, which was lower than in our study. The low bone 
mass was defined as T score lower than -1.5 , which was not in accordance with the WHO definition. These definitions were different than those used in our study, thus precluding comparison regarding the prevalence of osteopenia. Another study from Japan [25], on 144 men over 65 years who visited a dental clinic, found osteoporosis in 47 (33\%) and osteopenia in 42 (29\%) men, but the BMD test was different from the current study. It was measured at the calcaneus by ultrasound densitometry, thus, making the comparison to the results in our study inarticulate.

There is controversy regarding distal radius BMD. It can predict fractures in men [26, 27] and is rarely affected by osteoarthritis, as opposed to the spine and hip. Therefore, it may be a sensitive test for osteoporosis in men. Osteoporosis of DR alone was found in $15 \%$ of men aged 70 and older [28]. The Endocrine Society guidelines for osteoporosis in men suggested measuring DR DXA when the spine or hip BMD could not be interpreted, and for men with hyperparathyroidism or on anti-androgen therapy, but it was not recommended routinely [29]. The reason is that there is no scientific evidence showing that men with osteoporosis in the radius exclusively, respond to osteoporosis treatment. According to these guidelines, pharmacologic treatment is recommended for those had fragility fractures of the hip or vertebrae, a T score less than -2.5 in the spine, femoral neck, total hip, or a T score between -1 and -2.5 and a FRAX ${ }^{\oplus}$ ten year probability for any fracture $\geq 20 \%$ or 10 -year risk of the hip fracture $\geq 3 \%$. The AACE/ACE guidelines for the diagnosis and treatment of postmenopausal osteoporosis of 2016 [30] recommended pharmacologic treatment of patients with similar indications but included DR BMD. In the future, with more data, DR could be included in the guidelines for men. We analyzed our results with and without the DR BMD.

Osteopenia was found in over $60 \%$ of our study participants, and more than $10 \%$ diagnosed with osteoporosis. This is alarming as due to the lack of routine screening in the male population, many elderly physicians with osteopenia or osteoporosis would not be diagnosed and treated to prevent osteoporotic fractures.

There is a relative lack of epidemiological data and guidelines for osteoporosis screening in men as compared to women. The lifetime risk of osteoporotic fractures in men is estimated to be between $10 \%$ and $25 \%$, depending on the population studied, with men experiencing it around ten years later than women. As life expectancy is increasing for men more than women, the prevalence of osteoporotic fractures in men is expected to increase, making them an interesting group to study.

The prevalence of osteoporosis in Holocaust survivors is known to be significantly higher than that of the general population, due to nutritional deficits and lack of exercise and sunlight [31, 32]. There were five of the study participants who were Holocaust survivors. However, it is not possible to conclude the general population of Holocaust survivors because of the small number of Holocaust survivors in the study.

Our study has several limitations. First, the assumption was made, based on previous studies, that young hospital-based doctors have a deficiency of vitamin D when compared to communitybased doctors [9], and suboptimal vitamin D levels generally [7, 8]. It is not known whether the specific doctors participating in this study had low vitamin D levels throughout their careers. It was decided not to measure current vitamin $D$ levels at the time of the study, as they may be different from the earlier levels. Also, hypogonadism was one of the questions that were asked to calculate the FRAX ${ }^{\circledast}$ score, but the serum testosterone level was not examined in our study. 
Another limitation of this study is the specific study population. It consisted of physicians, and some factors that affect the risk of osteoporosis viz., non-smoking, exercise, and diet, including calcium in this specific population may counteract the effects of lack of vitamin $D$.

The third limitation is selection bias. The study had an approximately 50\% response-rate; as the healthier and more mobile subjects were included due to self-selection. This selection, however, presumptively enhanced the relevance of our results.

\section{Conclusions}

In our trial, we examined BMD in male hospitalists over the age of 65 . It seems that hospitalists have a high prevalence of osteoporosis and osteopenia. The data from this study may be useful for the decision of screening tests in this population, as well as designing larger confirmatory studies on the prevalence of osteopenia and osteoporosis in at-risk populations.

\section{Author Contributions}

NS collected part of the data and wrote the main manuscript of the article. CFS collected part of the data and summarized the data, MF analysed the data and wrote part of the main manuscript, GM proposed the initial idea of the trial, made critical revisions, and read and approved the final manuscript.

\section{Funding}

No funding was received for this trial.

\section{Competing Interests}

The authors have declared that no competing interests exist.

\section{References}

1. Holick MF. High prevalence of vitamin D inadequacy and implications for health. Mayo Clinic Proc. 2006; 81: 353-373.

2. Holick MF. Sunlight and vitamin D for bone health and prevention of autoimmune diseases, cancers and cardiovascular disease. Am J Clin Nutr. 2004; 80: 1678S-1688S.

3. Melamed ML, Muntner P, Michos ED. Serum 25-Hydroxyvitamin D levels and the prevalence of peripheral arterial disease - results from NHANES 2001-2004. Arterioscler Thromb Vasc Biol. 2008; 28: 1179-1185.

4. Keun N, Giovannucci E. Vitamin D supplements and cancer incidence and mortality: A metaanalysis. Br J Cancer. 2014; 111: 976-980.

5. Autier P, Gandini S. Vitamin D supplementation and total mortality, a meta-analysis of randomized controlled trials. Arch Intern Med. 2007; 167: 1730-1737.

6. Azizi E, Pavlotsky F, Vered I. Occupational exposure to solar UVB and seasonal monitoring of serum levels of 25-hydroxy vitamin D3: A case-control study. Photochem Photobiol. 2009; 85: 1240-1244. 
7. Haney EM, Stadler D, Bliziotes MM. Vitamin D insufficiency in internal medicine residents. Calcif Tissue Int. 2005; 76: 11-16.

8. Manickam B, Washington T, Villagrana N. Determinants of circulating 25-hydroxyvitamin D and bone mineral density in young physicians. Endocr Pract. 2012; 18: 219-226.

9. Munter G, Levi-Vineberg T, Sylvetsky N. Vitamin D deficiency among physicians: A comparison between hospitalists and community-based physicians. Osteoporos Int. 2015; 26:1673-1676.

10. Sowah D, Fan X, Dennett L, Hagtvedt R, Straube S. Vitamin D levels and deficiency with different occupations: A systematic review. BMC Public Health. 2017; 17: 519.

11. Watts NB. Osteoporosis in Men. Endocr Pract. 2013; 19: 834-838.

12. Berntsen GKR, Fønneb $\varnothing$ V , Tollan A. Forearm bone mineral density by age in 7,620 men and women the troms $\varnothing$ study, a population-based study. Am J Epidemiol. 2001; 153: 465-473.

13. Kanis JA, on behalf of the World Health Organization Scientific Group (2007). Assessment of osteoporosis at the primary health-care level. Technical Report. World Health Organization Collaborating Centre for Metabolic Bone Diseases, University of Sheffield, UK. 2007.

14. FRAX assessment at: https://www.sheffield.ac.uk/FRAX/tool.aspx?country=61.

15. Beaudoin C, Moore L, Gagné M, Bessette L, Ste-Marie LG, Brown JP, et al. Performance of predictive tools to identify individuals at risk of non-traumatic fracture: A systematic review, meta-analysis, and meta-regression. Osteoporos Int. 2019; 30: 721-740.

16. National Ostoporosis Foundation. 2013 Clinician's Guide to Prevention and Treatment of Osteoporosis. http://nof/public/content/resource/913/files/580.pdf.

17. International Society for Clinical Densitometry. 2013 ISCD Official Positions - Adult. At: www.iscd.org/official-positions/2013-iscd-official-positions-adult.

18. Papaioannou A, Morin S, Cheung AM, Atkinson S, Brown JP, Feldman S, et al. 2010 Clinical practice guidelines for the diagnosis and management of osteoporosis in Canada: Summary. CMAJ. 2010; 182: 1864

19. American Association of Clinical Endocrinologists Medical Guidelines for Clinical Practice for the Diagnosis and Treatment of Postmenopausal Osteoporosis. At: www.aace.com/pub/pdf/guidelines/OsteoGuidelines2010.pdf.

20. U.S. Preventive Services Task Force. Screening for Osteoporosis Recommendation Statement. At: www.uspreventiveservicestaskforce.org/uspstf10/osteoporosis/osteors.htm.

21. Cooper C, Campion G, Melton LJ. Hip fractures in the elderly: A world-wide projection. Osteoporos Int. 1992; 2: 285-289.

22. Cooper C, Atkinson EJ, O'Fallon WM, Melton $\sqcup$ 3rd. Incidence of clinically diagnosed vertebral fractures: A population based study in Rochester Minnesota, 1985-1989. J Bone Miner Res. 1992; 7: 221-227.

23. Wright NC, Looker AC, Saag KG, Curtis JR, Delzell ES, Randall S, et al. The recent prevalence of osteoporosis and low bone mass in the United States based on bone mineral density at the femoral neck or lumbar spine. J Bone Miner Res. 2014; 29: 2520-2526.

24. Gourlay ML, Overman RA, Fine JP, Filteau G, Cawthon PM, Schousboe JT, et al. Osteoporotic fractures in men (MrOS) research group: Time to osteoporosis and major fracture in older men: The MrOS study. Am J Prev Med. 2016; 50: 727-736.

25. Ohtsuki H, Kawakami M, Kawakami T, Takahashi K, Kirita T, Komasa Y. Risk of osteoporosis in elderly individuals attending a dental clinic. Int Dent J. 2017; 67: 117-122. 
26. Gärdsell $P$, Johnell $O$, Nilsson $B E$. The predictive value of forearm bone mineral content measurements in men. Bone. 1990; 11: 229-232.

27. Melton LJ 3rd, Atkinson EJ, O'Connor MK, O'Fallon WM, Riggs BL. Bone density and fracture risk in men. J Bone Miner Res. 1998; 13: 1915-1923.

28. Wiemann LM, Vallarta-Ast N, Krueger D, Binkley N. Effect of female database use for T-score derivation in men. J Clin Densitom. 2007; 10: 244-248.

29. Watts NB, Adler RA, Bilezikian JP, Drake MT, Eastell R, Orwoll ES, et al. Osteoporosis in men: An Endocrine Society clinical practice guideline. J Clin Endocrinol Metab. 2012; 97: 1802-1822.

30. Camacho PM, Petak SM, Binkley N, Clarke BL, Harris ST, Hurley DL, et al. American Association of Clinical Endocrinologists and American College of Endocrinology clinical practice guidelines for the diagnosis and treatment of postmenopausal osteoporosis. Endocr Pract. 2016; 22: 1111-1118.

31. Marcus EL, Menczel J. Higher prevalence of osteoporosis among female Holocaust survivors. Osteoporos Int. 2007; 18: 1501-1506.

32. Kueper J, Beyth S, Liebergall M, Kaplan L, Schroeder JE. Evidence for the adverse effect of starvation on bone quality: A review of the literature. Int J Endocrinol. 2015; 2015: 628740.

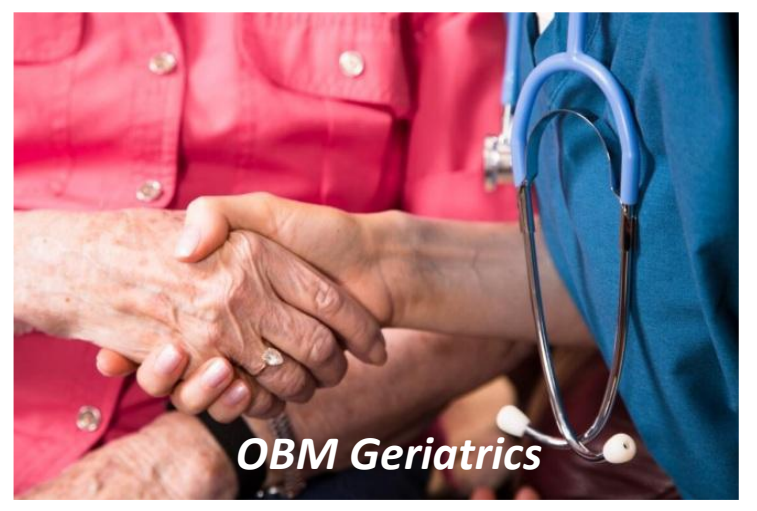

Enjoy $O B M$ Geriatrics by:

1. Submitting a manuscript

2. Joining in volunteer reviewer bank

3. Joining Editorial Board

4. Guest editing a special issue

For more details, please visit:

http://www.lidsen.com/journals/geriatrics 